1 Fundação de Apoio à Escola Técnica do Estado do Rio de Janeiro (Faetec) - Rio de Janeiro (RJ), Brasil. Fundação Oswaldo Cruz (Fiocruz), Instituto de Comunicação e Informação Científica e Tecnológica em Saúde (Icict), Programa de Pós-graduação em Informação e Comunicação em Saúde (PPGICS) - Rio de Janeiro (RJ), Brasil. rpprjbr@gmail.com

2 Fundação Oswaldo Cruz (Fiocruz), Escola Nacional de Saúde Pública Sergio Arouca (Ensp) - Rio de Janeiro (RJ), Brasil. andrepereira@ensp.fiocruz.br

${ }^{3}$ Fundação Oswaldo Cruz (Fiocruz), Instituto de Comunicação e Informação Científica e Tecnológica em Saúde (lcict), Programa de Pós-graduação em Informação e Comunicação em Saúde (PPGICS) - Rio de Janeiro (RJ), Brasil. rafaelaluzia@gmail.com

\section{Avaliação da qualidade da informação em sites de tuberculose: análise de uma experiência participativa}

\author{
Information quality assessment on tuberculosis sites: analysis of a \\ participatory experience
}

Rodolfo Paolucci', André Pereira Neto ${ }^{2}$, Rafaela Luzia ${ }^{\mathbf{3}}$

RESUMO As informações de saúde encontradas na Internet são, muitas vezes, incorretas, incompletas ou incompreensíveis para a população, oferecendo riscos aos cidadãos e à coletividade. Diferentes iniciativas têm sido adotadas para sanar esse problema. Este artigo analisa uma experiência de avaliação da qualidade da informação em sites de tuberculose, desenvolvida por laboratório da Fundação Oswaldo Cruz. Metodologicamente, discute seus resultados à luz de duas revisões sistemáticas. Apesar do número reduzido de sites avaliados e da limitada participação de usuários e profissionais, a experiência revela que o problema da qualidade da informação de saúde on-line afeta sites públicos e privados.

PALAVRAS-CHAVE Avaliação em saúde. Internet. Informação de saúde ao consumidor. Participação social. Tuberculose.

\begin{abstract}
Health information found on the Internet is often inaccurate, incomplete or incomprehensible by the population, posing risks to citizens and collectivities. Different initiatives have been made to remedy this problem. This article analyzes an evaluation experience of information quality on tuberculosis sites, developed by a laboratory of the Oswaldo Cruz Foundation. Methodologically, it discusses the results in light of two systematic reviews. Despite the reduced number of sites and the limited participation of users and professionals, this experience shows that quality problem of online health information affects public and private sites.
\end{abstract}

KEYWORDS Health evaluation. Internet. Consumer health information. Social participation. Tuberculosis. 


\section{Introdução}

A Internet permite que o cidadão, com pouca habilidade para manipular dispositivos eletrônicos e baixo poder aquisitivo para adquiri-los, acesse uma quantidade incomensurável de informações (SILVEIRA; COSTA; LIMA, 2012). Além de acessar, ele pode produzir, compartilhar e disseminar informações para qualquer pessoa, a qualquer hora e de qualquer lugar com conexão ao World Wide Web (Web) (SOUZA; LUZ; RAbelLo, 2008). Por essa razão, a cada dia se produzem novos sites, blogs, páginas e facebooks sobre os mais variados temas e problemas.

A saúde é um dos temas mais acessados, postados e compartilhados na Internet. $\mathrm{O}$ Comitê Gestor de Internet no Brasil (CGI), órgão interministerial do governo federal, realiza periodicamente pesquisas nacionais para acompanhar a expansão da Internet em nosso País. A última pesquisa, realizada em 2013, indicou que a procura por informações e serviços de saúde é o segundo maior interesse dos internautas brasileiros, independente da renda, grau de instrução e região do País em que residam (BARBOSA, 2014). Tal interesse talvez esteja associado ao fato de a saúde ter se tornado nos últimos anos uma das principais preocupações da humanidade e se transformado em um "valor supremo" (FUREDI, 2006, P. 14).

E quem produz as informações de saúde?

Graças à liberdade inerente à Internet, qualquer pessoa pode produzir informação. Na saúde, podemos encontrar ambientes virtuais construídos por instituições públicas e privadas de ensino e pesquisa, agências governamentais e não governamentais, associações de pacientes e de profissionais e pelos próprios indivíduos. Essa possibilidade permite que as informações sejam postadas e compartilhadas sem qualquer tipo de avaliação. Tal condição possibilita que sejam disponibilizadas informações, muitas vezes, incompletas, contraditórias, incorretas ou até fraudulentas (PEREIRA NETO ET AL., 2013). Além disso, podem estar corretas e atualizadas, mas serem apresentadas de maneira que dificulte sua compreensão pelo cidadão (PEREIRA NETO; PAOLUCCI, 2014).

Estudos distintos evidenciaram que a informação on-line é, na maioria das vezes, insuficiente ou insatisfatória (BARBOSA; MARTINS, 2007; CUBAS; FELCHNER, 2012; DEL GIGLIO ET AL., 2012; GUARDIOLA-WANDEN-BERGHE， SANZ-VALERO; WANDENBERGHE, 2012; SILVA; GUBERT, 2010; SILVEIRA, COSTA; LIMA, 2012; SOUZA, LUZ; RABELLO, 2008). Informações como essas podem ter graves consequências para o cidadão e a coletividade, dentre as quais destacam-se as confusões com sintomas, os atrasos nos diagnósticos, as implicações nos tratamentos e a automedicação sem orientação.

Então, como o problema da (má) qualidade da informação de saúde disponível na Internet tem sido enfrentado?

A avaliação da qualidade da informação de saúde na Internet é uma possibilidade para tratar esse problema. Lopes (2012) analisou treze das principais iniciativas internacionais que visam a avaliar a qualidade da informação em saúde na Internet. Elas são provenientes de organismos internacionais, instituições públicas, privadas e não governamentais. Segundo a autora, os diversos critérios de qualidade empregados nas avaliações foram propostos para resguardar os usuários de informações que ofereçam riscos à saúde. Algumas instituições, ao final de uma avaliação, oferecem um selo de qualidade para ser exposto no site. Outras fornecem instruções para que os provedores de informação sigam ao disponibilizar seus conteúdos. Há, ainda, aquelas que divulgam um código de conduta para orientar o usuário a verificar se os sites estão em conformidade ou não com critérios preestabelecidos (MENDONÇA; PEREIRA NETO, 2015).

A recente revisão sistemática realizada por Paolucci (2015) indica o crescimento da produção acadêmica internacional sobre avaliação da qualidade da informação de saúde na Internet nos últimos anos. Nesse estudo, foram encontrados apenas sete 
trabalhos em 2001; 22 em 2011; e 46 em 2013. Nesse sentido, podemos afirmar que esse campo de estudos está em expansão.

E no Brasil, existem iniciativas institucionais e pesquisas acadêmicas na área da avaliação da qualidade da informação de saúde na Internet?

Em termos institucionais, destaca-se o papel do Conselho Regional de Medicina de São Paulo (Cremesp), que publicou, em 2001, uma Resolução acompanhada do 'Manual princípios éticos para sites de medicina e saúde na internet' (CREMESP, 2016). Essa Resolução apresenta recomendações para usuários e gestores de sites e determina que os sites de médicos e instituições ligadas ao Cremesp estejam em conformidade com a Resolução.

Em termos acadêmicos, a produção bibliográfica brasileira sobre o tema da avaliação da qualidade da informação de saúde na Internet parece incipiente. A partir do levantamento bibliográfico realizado por Paolucci (2015) sobre o tema na Scientific Electronic Library Online (SciELO), foram encontrados 19 artigos dedicados especificamente à avaliação de qualidade da informação. A produção é dispersa em periódicos dos campos biomédicos e das ciências sociais e saúde. Em geral, os estudos avaliaram a qualidade da informação de uma patologia específica, de um comportamento ou de um estilo de vida associado à saúde ou à doença, como o aleitamento materno e o tabagismo. Na maioria dos casos, os autores utilizaram os critérios adotados pelas iniciativas internacionais analisadas por Lopes (2012) e Mendonça e Pereira Neto (2015). Eles exerceram, ainda, os papéis de avaliadores das informações ao verificarem a conformidade dos sites com esses critérios.

O Laboratório Internet, Saúde e Sociedade (LaISS), vinculado ao Centro de Saúde Escola Germano Sinval de Faria da Escola Nacional de Saúde Pública Sergio Arouca (Ensp) da Fundação Oswaldo Cruz (Fiocruz), desenvolve, desde 2012, atividades de avaliação da qualidade da informação de saúde na Internet. Sua primeira experiência nesse sentido avaliou a qualidade da informação de sites de dengue (PEREIRA NETO; PAOLUCCI, 2014). Uma segunda, realizada em 2014, avaliou a qualidade da informação em sites de tuberculose (TB).

Nos dois casos, realizou-se 'pesquisa participativa'. Para tanto, foi necessária a mudança na postura do pesquisador e dos pesquisados, porque, nesse tipo de investigação, todos são coautores do processo de diagnóstico da situação-problema e da construção de vias que possam resolver as questões (OLIVEIRA; OLIVEIRA, 1985). Nesse caso, os participantes usuários e profissionais tiveram a oportunidade de discutir e apresentar propostas na elaboração dos critérios e indicadores utilizados na avaliação. Além disso, eles foram os avaliadores. Houve preocupação em manter as diferenças entre esses segmentos sem hierarquizá-los (PASSOS ET AL., 2013). As contribuições foram incorporadas e debatidas exaustivamente.

Este artigo pretende contribuir com esse campo de avaliação em saúde por meio da análise da experiência de avaliação da qualidade da informação em sites de tuberculose realizada em 2014.

\section{Métodos}

Como mencionamos anteriormente, a avaliação da qualidade da informação de saúde na Internet tem sido objeto de interesse acadêmico há alguns anos. Dois estudos de revisão sistemática sobre o tema reuniram a produção acadêmica disponível em diferentes bases bibliográficas internacionais. O primeiro estudo identificou e analisou 79 artigos publicados até o ano de 2001 (EYSENBACH ET AL., 2002). O segundo estudo atualizou o primeiro. Obedecendo às mesmas diretrizes para realização de revisões sistemáticas, definidas pela The Cochrane Collaboration, foram identificados e analisados 279 artigos publicados entre os anos de $2001 \mathrm{e}$ 
2014, disponíveis em bases bibliográficas internacionais (PAOLUCCI, 2015).

Nas duas revisões sistemáticas, percebemos que os estudos dedicados à avaliação da qualidade da informação em sites de saúde organizam essa atividade em três momentos: seleção do(s) de avaliador(es), construção dos critérios e indicadores de qualidade, seleção dos sites avaliados.

Neste artigo, informaremos como foi realizada a experiência de avaliação da qualidade da informação em sites de tuberculose.

\section{Seleção dos avaliadores}

O trabalho de Eysenbach et al. (2002) não verificou como foi a seleção dos avaliadores nos estudos analisados. Entretanto, dentre suas conclusões, consta que "nenhum dos estudos conduziu testes de compreensão com consumidores reais ou usou o julgamento de especialistas em alfabetização" (EYSENBACH ET AL., 2002, P. 2695).

Nos estudos identificados no trabalho de Paolucci (2015), os avaliadores dos sites de saúde foram divididos em três categorias: 'autor' (79\%), quando os avaliadores são os próprios autores do artigo; 'especialista' (9\%), quando os avaliadores são especialistas nos temas dos sites avaliados ou convidam algum especialista para exercer o papel de avaliador; e 'usuário' (5\%), quando os avaliadores são usuários das informações disponíveis nos sites. Paolucci (2015) conclui afirmando que

a realidade observada naquela época sobre os avaliadores de sites de saúde ainda é atual, mesmo com esta participação inexpressiva de Especialistas e Usuários. (PAOLUCCI, 2015, P. 74).

Além disso, os estudos em que houve participação de 'especialistas' e de 'usuários' não seguiram qualquer padrão, pois o número de envolvidos de cada grupo e os métodos empregados foram distintos (PAOLUCCI, 2015).

A experiência de avaliação da qualidade da informação em sites de tuberculose, analisada neste artigo, contou com a participação de moradores das comunidades do Complexo de Manguinhos e de profissionais de saúde que atuam na atenção primária ou que pesquisam tuberculose.

O primeiro grupo é formado por dezesseis mulheres e quatro homens com níveis variados de escolaridade e idades entre 30 e 60 anos, residentes nas comunidades de Manguinhos e usuários dos serviços de atenção primária do Centro de Saúde Escola Germano Sinval de Faria (CSEGSF /Ensp/ Fiocruz). Eles representam os usuários do Sistema Único de Saúde (SUS) na avaliação dos sites de tuberculose. Foram convidados porque haviam participado de uma atividade de inclusão digital e da primeira experiência de avaliação da qualidade de sites de saúde relacionada com a dengue (PEREIRA NETO; PAOLUCCI, 2014). Naquela primeira experiência, muitos deles não tinham habilidades e conhecimentos básicos de informática. Nesta experiência de avaliação de sites de tuberculose, eles demonstraram maior desenvoltura no uso dos computadores. Os vinte moradores do complexo de Manguinhos a participa da pesquisa participativa receberam um auxílio de R\$ 100,00 por mês para atuarem como avaliadores representantes dos usuários do SUS graças ao apoio que o laboratório recebeu da Vice-Presidência de Ambiente, Atenção e Promoção da Saúde. Esse apoio elevou a autoestima do grupo e contribuiu para seu engajamento.

O Complexo de Manguinhos é composto por treze comunidades: Vila União, Parque João Goulart, Vila Turismo, CHP-2, Parque Carlos Chagas, Vitória de Manguinhos, Mandela de Pedra, Conjuntos Ex-Combatentes e Suburbana, Conjunto Nelson Mandela, Conjunto Samora Machel, Parque Oswaldo Cruz, Vila São Pedro e Comunidade Agrícola de Higienópolis (BORGES, 2007).

Nessas comunidades, as condições socioeconômicas não são uniformes. As condições de vida e os indicadores sociais revelam 
grandes contrastes entre diferentes realidades em Manguinhos. Entretanto, segundo Borges (2007), alguns aspectos dessas comunidades se manifestam de forma semelhante. Para a autora, a maioria da população do Complexo sofre com os baixos níveis de escolaridade, sendo a vida desses habitantes delineada pelas péssimas oportunidades empregatícias.

Essa região também é marcada pelas condições de habitação inadequadas. Em geral, não possui saneamento básico, o ambiente é insalubre e as oportunidades culturais e de lazer são praticamente inexistentes (BORGES, 2007). Fernandes e Costa (2013) destacam, também, que a região possui graves problemas ambientais advindos

tanto das fábricas como das moradias, nos rios que cortam a região, além da proximidade com a Avenida Brasil e a Refinaria de Petróleos de Manguinhos S.A. (FERNANDES; COSTA, 2013, P. 123).

Outro ponto crucial destacado por Borges (2007) é o alto índice de violência e tráfico de drogas presente em grande parte do Complexo. Vale ressaltar que, dentre 126 grupos de bairros da cidade do Rio, Manguinhos apresenta o quinto pior Índice de Desenvolvimento Humano (IDH), seguido pelo Jacarezinho, em sexto (WIKIRIO, 2016).

O segundo grupo é composto por 19 profissionais de saúde. Foi possível sensibilizar e envolver, nesse trabalho de avaliação, cinco profissionais que participaram da primeira experiência de avaliação sobre dengue e que têm vínculo com o Centro de Saúde Escola Germano Sinval de Faria. A esse grupo, juntaram-se quatro profissionais do Centro de Referência Professor Hélio Fraga (CRPHF/Ensp/Fiocruz), departamento da Ensp dedicado à pesquisa e assistência em tuberculose, três estudantes do curso de Residência Multiprofissional em Saúde da Família (Ensp/Fiocruz), uma pesquisadora do Instituto Oswaldo Cruz (IOC/Fiocruz) e seis pesquisadores que desenvolviam trabalho de investigação sobre tuberculose no Laboratório de Situações Endêmicas Regionais (Laser/Ensp/Fiocruz). Todos participaram como voluntários avaliando as informações dos sites de tuberculose.

Nesse sentido, podemos constatar que a experiência relatada neste artigo contou com uma equipe de avaliadores composta por 20 usuários: moradores das comunidades de Manguinhos e 19 especialistas, profissionais e pesquisadores de diferentes órgãos da Fiocruz. Essa avaliação participativa foi observada em apenas 2,5\% das experiências identificadas por Paolucci (2015).

\section{Construção dos critérios e indicadores de qualidade}

A revisão sistemática realizada por Eysenbach et al. (2002) identificou 79 artigos que avaliaram a qualidade da informação em sites de saúde. Nesses estudos, foram encontrados 86 critérios distintos para realizar a avaliação. Eysenbach et al. (2002) agruparam esses critérios em cinco, que foram denominados e definidos da seguinte forma: 'acurácia' - mede o grau de concordância da informação com a melhor evidência médica ou aquela que é geralmente aceita; 'legibilidade' - objetiva verificar o nível de compreensão da informação disponibilizada; 'abrangência' - verifica se as informações disponíveis abrangem todos os aspectos relevantes daquele tema ou problema; 'design' - preocupa-se com o aspecto visual ou estético de um site, como o layout, a apresentação de sua interface e os recursos disponíveis para a navegação. Inclui, portanto, as dimensões de usabilidade e acessibilidade. Foi definido, ainda, o critério 'técnico' - avalia como a informação é apresentada, identificando se o site apresenta a autoria, atribuição, divulgação, atualização e patrocínio subjacentes à informação disponibilizada. Cada critério era constituído por um número diferente de indicadores - perguntas específicas que servem para medir o grau de conformidade de cada critério. 
A segunda revisão sistemática, realizada por Paolucci (2015), procurou verificar se esses cinco critérios continuaram sendo utilizados nos estudos publicados entre 2001 e 2014. Pretendeu verificar, ainda, a incidência de cada um deles. Paolucci (2015) evidenciou que os cinco critérios continuaram sendo adotados (PAOLUCCI, 2015). Do ponto de vista estatístico, os critérios mais utilizados foram respectivamente 'acurácia' (64\%), 'legibilidade' (38\%), 'abrangência' (27\%), 'técnico' (24\%) e 'design' (17\%).

Assim, a segunda revisão sistemática constatou que os critérios identificados nos estudos analisados na primeira revisão foram mantidos. Portanto, o quadro metodológico de avaliação de sites de saúde continuou sendo, em linhas gerais, o mesmo.

A experiência de avaliação da qualidade da informação em sites de tuberculose utilizou os mesmos cinco critérios, embora tenha apresentado pequena diferença em relação ao critério 'design', que merece ser mencionada.

Nessa experiência, o critério 'design' se restringiu ao domínio da 'interatividade'. Assim, a equipe pretendeu avaliar como a comunicação entre os usuários e os gestores do site poderia ser estabelecida.

Essa decisão esteve amparada em uma das características que distingue o meio de comunicação virtual dos tradicionais: a capacidade de os usuários trocarem informações entre si e com os gestores de plataformas na Internet. Essa característica foi valorizada pela equipe da pesquisa ao introduzir tal dimensão na avaliação. Sendo assim, um site de saúde deve oferecer condições para que os usuários consigam, por exemplo, compartilhar informações, tirar dúvidas e apresentar elogios ou queixas ao gestor do site.

A inclusão da dimensão foi fruto do processo de construção dos critérios utilizados, sendo debatidos pelos participantes da avaliação, quer sejam moradores de Manguinhos ou especialistas em tuberculose.

Em relação ao primeiro grupo, foram constituídas duas turmas que se reuniram semanalmente durante sete meses.
As discussões ampararam-se no processo de comunicação dialógica (FREIRE, 1971). Foram promovidas discussões para compreensão de cada conceito ligado ao mundo virtual e, em seguida, para o desenvolvimento dos critérios e indicadores. Qualquer tomada de decisão sobre a inclusão de algum item ou de como ele deveria ser redigido foi precedida de intenso debate entre os pesquisadores cidadãos. Eles participaram da construção dos critérios e dos indicadores de 'legibilidade', 'abrangência', 'técnico' e 'design'.

Em relação ao segundo grupo, a equipe que coordenou a avaliação estreitou seus laços de trabalho com uma equipe de profissionais do Centro de Referencia Professor Hélio Fraga. Com eles, foram construídos os indicadores de acurácia.

Cabe destacar que os resultados dessa avaliação foram contabilizados por meio de critérios construídos por processo participativo. Em seguida, foi construído, com os mesmos usuários e especialistas, um número diferente de indicadores para cada critério, a saber: 'técnico', oito indicadores; 'interatividade', cinco indicadores; 'abrangência', oito indicadores; 'legibilidade', 20 indicadores; 'acurácia', 21 indicadores. Cada indicador foi apresentado na forma de pergunta avaliativa com as opções SIM ou NÃO, onde apenas uma foi considerada ideal. Por exemplo, dentre os indicadores do critério 'abrangência' constou a seguinte pergunta: 'Tem informação sobre prevenção?'. A resposta ideal é SIM. No critério 'legibilidade', consta a seguinte pergunta: 'Você teve dificuldade de entender a informação sobre prevenção?’. A resposta ideal é NÃO. Somente os indicadores do critério 'acurácia' foram apresentados na forma de sentenças afirmativas que continham as opções de respostas 'Completa', 'Incompleta', 'Ausente' e 'Incorreta'. Por exemplo, um indicador de 'acurácia' foi: 'A vacinação da BCG é obrigatória para todas as crianças e é oferecida pela rede pública'. A resposta ideal neste indicador é a 'Completa'. Os 62 indicadores encontram-se no quadro 1 apresentado a seguir. 
Quadro 1. Indicadores de qualidade por critério

\begin{tabular}{|c|c|}
\hline Critério & Indicador \\
\hline \multirow{8}{*}{ TÉCNICO } & Constam informações sobre o responsável ou Instituição? \\
\hline & Há quanto tempo foi feita a última ATUALIZAÇÃO? \\
\hline & O site tem alguma PROPAGANDA comercial? \\
\hline & Existe a fonte de informação para PREVENÇÃO? \\
\hline & Existe a fonte de informação para TRANSMISSÃO? \\
\hline & Existe a fonte de informação para SINTOMAS? \\
\hline & Existe a fonte de informação para DIAGNÓSTICO? \\
\hline & Existe a fonte de informação para TRATAMENTO? \\
\hline \multirow{5}{*}{ INTERATIVIDADE } & Existe um CANAL DE COMUNICAÇÃO para contato? \\
\hline & Participa de alguma REDE SOCIAL? \\
\hline & Existe ferramenta de busca, de pesquisa? \\
\hline & Existe um MENU principal? \\
\hline & A PRIMEIRA PÁGINA do site é ATRAENTE? \\
\hline \multirow{8}{*}{ ABRANGÊNCIA } & Existe informação sobre PREVENCCÃO? \\
\hline & Existe informação sobre TRANSMISSÃO? \\
\hline & Existe informação sobre SINTOMAS? \\
\hline & Existe informação sobre DIAGNÓSTICO? \\
\hline & Existe informação sobre ONDE fazer o DIAGNÓSTICO? \\
\hline & Existe informação sobre TRATAMENTO? \\
\hline & Existe informação sobre ONDE fazer o TRATAMENTO? \\
\hline & Existe informação sobre os EFEITOS COLATERAIS do TRATAMENTO? \\
\hline \multirow{17}{*}{ LEGIBILIDADE } & Você teve dificuldade de entender a informação sobre PREVENÇÃO? \\
\hline & Você encontrou PALAVRAS que não conhecia na página de PREVENÇÃO? \\
\hline & Existem IMAGENS na página de PREVENÇÃO? \\
\hline & As IMAGENS na página de PREVENÇÃO ajudam a entender o texto? \\
\hline & Você teve dificuldade de entender a informação sobre TRANSMISSÃO? \\
\hline & Você encontrou PALAVRAS que não conhecia na página de TRANSMISSÃO? \\
\hline & Existem IMAGENS na página de TRANSMISSÃO? \\
\hline & As IMAGENS na página de TRANSMISSÃO ajudam a entender o texto? \\
\hline & Você teve dificuldade de entender a informação sobre SINTOMAS? \\
\hline & Você encontrou PALAVRAS que não conhecia na página de SINTOMAS? \\
\hline & Existem IMAGENS na página de SINTOMAS? \\
\hline & As IMAGENS na página de SINTOMAS ajudam a entender o texto? \\
\hline & Você teve dificuldade de entender a informação sobre DIAGNÓSTICO? \\
\hline & Você encontrou PALAVRAS que não conhecia na página de DIAGNÓSTICO? \\
\hline & Existem IMAGENS na página de DIAGNÓSTICO? \\
\hline & As IMAGENS na página de DIAGNÓSTICO ajudam a entender o texto? \\
\hline & Você teve dificuldade de entender a informação sobre TRATAMENTO? \\
\hline
\end{tabular}


Quadro 1. (cont.)

\begin{tabular}{|c|c|}
\hline \multirow{3}{*}{ LEGIBILIDADE } & Você encontrou PALAVRAS que não conhecia na página de TRATAMENTO? \\
\hline & Existem IMAGENS na página de DIAGNÓSTICO? \\
\hline & As IMAGENS na página de TRATAMENTO ajudam a entender o texto? \\
\hline \multirow{26}{*}{ ACURÁCIA } & PREVENÇÃO \\
\hline & A Tuberculose pode ser prevenida em lugares arejados. \\
\hline & $\begin{array}{l}\text { A vacina BCG protege as crianças das formas mais graves da tuberculose, como, por exem- } \\
\text { plo, da meningite tuberculosa. }\end{array}$ \\
\hline & A vacinação da BCG é obrigatória a todas as crianças e é oferecida pela rede pública. \\
\hline & TRANSMISSÃO \\
\hline & $\begin{array}{l}\text { A tuberculose é transmitida por via aérea, através da tosse, fala ou espirro, e não por contato } \\
\text { físico, como, por exemplo, aperto de mão, ou com utensílios domésticos. }\end{array}$ \\
\hline & A tuberculose pulmonar e laríngea são as que transmitem a doença. \\
\hline & $\begin{array}{l}\text { Na maioria das vezes, se o tratamento for realizado corretamente, em } 15 \text { dias, o doente não } \\
\text { transmite mais. }\end{array}$ \\
\hline & $\begin{array}{l}\text { O paciente que apresenta a o exame de escarro negativo, ou baciloscopia negativa, não } \\
\text { transmite a doença. }\end{array}$ \\
\hline & SINTOMAS \\
\hline & O principal sintoma da tuberculose é a tosse por mais de três semanas. \\
\hline & $\begin{array}{l}\text { Febre, perda de apetite, perda de peso, suor noturno e escarro com sangue são sintomas fre- } \\
\text { quentes da tuberculose: até dois sintomas=Incompleto; três ou mais sintomas= Completo. }\end{array}$ \\
\hline & $\begin{array}{l}\text { Os sintomas desaparecem com o uso regular das medicações. Isso não significa que o pa- } \\
\text { ciente esteja curado. }\end{array}$ \\
\hline & DIAGNÓSTICO \\
\hline & $\begin{array}{l}\text { Quando se divide o ambiente com alguém com tuberculose é necessário realizar uma avalia- } \\
\text { ção de saúde. }\end{array}$ \\
\hline & $\begin{array}{l}\text { A prova tuberculínica (PPD) é o teste diagnóstico para saber se o indivíduo teve contato com } \\
\text { o bacilo. }\end{array}$ \\
\hline & O indivíduo pode se infectar pelo bacilo e não desenvolver a doença. \\
\hline & $\begin{array}{l}\text { Todo paciente com tuberculose deve fazer o teste anti-HIV, pois o portador de HIV/Aids tem } \\
\text { maior chance de desenvolver a tuberculose. }\end{array}$ \\
\hline & $\begin{array}{l}\text { O principal exame para diagnosticar a tuberculose pulmonar é o exame de escarro, ou baci- } \\
\text { loscopia. }\end{array}$ \\
\hline & O Raio X de tórax é um exame que auxilia no diagnóstico da tuberculose pulmonar. \\
\hline & TRATAMENTO \\
\hline & A tuberculose tratada corretamente tem cura. \\
\hline & A duração do tratamento é de no mínimo seis meses. \\
\hline & $\begin{array}{l}\text { O paciente que abandonar ou usar os medicamentos irregularmente não será curado. A } \\
\text { doença pode piorar e o bacilo pode tornar-se resistente aos medicamentos. }\end{array}$ \\
\hline & Os medicamentos são gratuitos e distribuídos na rede pública. \\
\hline & $\begin{array}{l}\text { Em caso de efeito colateral, o medicamento só deverá ser suspenso por orientação do pro- } \\
\text { fissional da saúde. }\end{array}$ \\
\hline
\end{tabular}


Os resultados da avaliação foram consolidados em números relativos. Esses percentuais indicam níveis de conformidade em cada indicador do site avaliado. Retomando o exemplo mencionado anteriormente, ao avaliar determinado site, se doze usuários não encontrassem dificuldade em compreender a informação sobre prevenção, este site estaria, nesse indicador, com $60 \%$ de conformidade. A média de todos os indicadores oferece o grau de conformidade naquele critério. A média de todos os critérios fornece o grau de conformidade do site. Assim, foi possível estabelecer ranking entre os sites. No site do LaISS (www.ensp.fiocruz. br/laiss), é possível consultar os resultados completos por grupo de avaliadores, por site e por critério.

Cabe mencionar, ainda, que o critério ‘acurácia' foi avaliado pelos 'usuários' e pelos 'especialistas'. O grau de conformidade nesse critério foi obtido por meio da média das avaliações dos dois grupos de avaliadores. Para explorarmos a relação entre as duas perspectivas de avaliação, calculamos o 'coeficiente de correlação' das respostas oferecidas a todos os indicadores do critério para cada site avaliado. Segundo Agresti e Finlay (2012), o 'coeficiente de correlação' representa a força da associação entre dados numéricos e uma possível tendência.

\section{Seleção dos sites avaliados}

Os estudos de avaliação da qualidade da informação em sites de saúde publicados até 2001 utilizaram motores de busca, catálogos ou listas disponíveis para selecionar sua base empírica (EYSENBACH ET AL., 2002). No estudo de Paolucci (2015), as estratégias de seleção foram divididas em dois tipos: 'motor de busca', quando um estudo usou o Google, Yahoo, Bing ou Ask para realizar a seleção; e 'arbitrário', quando o estudo selecionou um ou mais sites segundo algum critério de inclusão justificado. Paolucci (2015) revelou que $84 \%$ dos estudos relacionados em sua revisão sistemática selecionaram os sites utilizando um 'motor de busca'.

A experiência de avaliação da qualidade da informação em sites de tuberculose combinou as duas estratégias: 'motor de busca' e 'arbitrária'. Na primeira, foram identificados os sites sobre tuberculose predominantes no 'motor de busca' Google. Neste trabalho, contou-se com a participação de dois estagiários de nível médio, integrantes da equipe da pesquisa. Cada estagiário pesquisou no Google utilizando a palavra-chave 'tuberculose' em 20 computadores diferentes localizados nas residências e lanhouses das comunidades de Manguinhos e anotou os dez primeiros sites apresentados na primeira página. As listas dos dez primeiros sites dos quarenta computadores foram reunidas. As recorrências dos sites na ordem em que apareceram nos resultados do Google foram calculadas. A maioria dos sites esteve presente em mais de um computador. Por essa razão, o número de sites selecionados foi menor que dez, quantidade máxima de resultados na primeira página do Google. Como foram excluídos endereços eletrônicos que não conduziam a um site ou não continham informação sobre TB passível de avaliação, a equipe da pesquisa chegou a uma lista contendo sete sites: dois vinculados a órgãos públicos, um de produção coletiva e quatro de iniciativa privada.

$\mathrm{Na}$ segunda estratégia, foi considerada a indicação de sites recomendados pela equipe de profissionais de saúde do Centro de Referência Professor Hélio Fraga. Por meio do método 'arbitrário', essa estratégia permitiu a inclusão dos sites ou páginas governamentais das cidades brasileiras com maior incidência da doença (Manaus, Porto Alegre e Rio de Janeiro) (BRASIL, 2013), além do site da Sociedade Brasileira de Pneumologia e Tisiologia e o do Programa Nacional de Controle da Tuberculose, vinculado ao Ministério da Saúde. Constituiu-se, assim uma amostra composta por doze sites (quadro 2 apresentado a seguir). 
Quadro 2. Amostra de sites selecionados

\begin{tabular}{ll}
\hline Site & Endereço eletrônico \\
\hline Sites sobre Tuberculose encontrados no Google em computadores de Manguinhos (RJ) \\
\hline Wikipédia & pt.wikipedia.org/wiki/Tuberculose \\
Brasil Escola & www.brasilescola.com/doencas/tuberculose.htm \\
Secretaria Estadual de Saúde do Paraná & www.saude.pr.gov.br/modules/conteudo/conteudo.php?conteudo=939 \\
Dr. Drauzio Varella & drauziovarella.com.br/letras/t/tuberculose/ \\
Minha Vida & www.minhavida.com.br/saude/temas/tuberculose \\
Secretaria Estadual de Saúde de São & www.saude.sp.gov.br/ses/perfil/cidadao/temas-de-saude/tuberculose/o- \\
Paulo & -que-e-tuberculose \\
G1 (Globo) & www.saude.sp.gov.br/ses/perfil/cidadao/temas-de-saude/tuberculose/o- \\
\hline Sites sugeridos por profissionais do CRPHF/Ensp/Fiocruz \\
\hline Prefeitura de Porto Alegre & www2.portoalegre.rs.gov.br/sms/default.php?p_secao=704 \\
Prefeitura do Rio de Janeiro & www.rio.rj.gov.br/web/sms/exibeconteudo?id=4277320 \\
Fundação de Medicina Tropical (AM) & www.fmt.am.gov.br/manual/tuberculose.htm \\
$\begin{array}{l}\text { Sociedade Brasileira de Pneumologia e } \\
\text { Tisiologia }\end{array}$ & www.sbpt.org.br/tuberculose-12111211/ \\
Programa Nacional de Controle da Tu- & www.blogdatuberculose.blogspot.com.br/p/conhecendo-tuberculose.html \\
berculose & \\
\hline
\end{tabular}

Os resultados da avaliação da qualidade da informação encontrada nesses sites serão apresentados e discutidos a seguir.

\section{Resultados e discussão}

Os resultados da experiência de avaliação da qualidade da informação de tuberculose na Internet indicam que nenhum dos sites avaliados obteve mais de $65 \%$ de conformidade com os critérios e os indicadores utilizados (tabela 1 destacado a seguir). Apenas um deles atingiu $50 \%$ de conformidade no critério 'legibilidade'. Ficamos surpresos com o fato de o site da Fundação de Medicina Tropical e da Sociedade Brasileira de Pneumologia e Tisiologia terem atingido os piores índices nesse critério. Duas das mais importantes e respeitadas instituições científicas de tuberculose oferecem informações que os usuários moradores de Manguinhos tiveram enormes dificuldades de compreender. 
Tabela 1. Ranking dos sites de tuberculose

\begin{tabular}{|c|c|c|c|c|c|c|c|c|c|}
\hline \multirow{2}{*}{ Posição } & \multirow[t]{2}{*}{ Site } & \multirow{2}{*}{ Técnico } & \multirow{2}{*}{ Interatividade } & \multirow{2}{*}{ Abrangência } & \multirow{2}{*}{ Legibilidade } & \multicolumn{3}{|c|}{ Acurácia } & \multirow{2}{*}{ Média Final } \\
\hline & & & & & & Usuários & Especialistas & Média & \\
\hline 1 & $\begin{array}{l}\text { Secretaria Estadual de Saúde } \\
\text { de São Paulo }\end{array}$ & $44 \%$ & $77 \%$ & $83 \%$ & $41 \%$ & $72 \%$ & $71 \%$ & $72 \%$ & $63 \%$ \\
\hline 2 & $\begin{array}{l}\text { Programa Nacional de Con- } \\
\text { trole de Tuberculose }\end{array}$ & $38 \%$ & $76 \%$ & $74 \%$ & $41 \%$ & $79 \%$ & $69 \%$ & $74 \%$ & $61 \%$ \\
\hline 3 & Minha Vida & $51 \%$ & $87 \%$ & $68 \%$ & $53 \%$ & $29 \%$ & $55 \%$ & $42 \%$ & $60 \%$ \\
\hline 4 & Prefeitura do Rio de Janeiro & $52 \%$ & $79 \%$ & $66 \%$ & $40 \%$ & $43 \%$ & $51 \%$ & $47 \%$ & $57 \%$ \\
\hline 5 & Wikipédia & $66 \%$ & $59 \%$ & $69 \%$ & $38 \%$ & $31 \%$ & $60 \%$ & $46 \%$ & $56 \%$ \\
\hline 6 & Prefeitura de Porto Alegre & $30 \%$ & $76 \%$ & $67 \%$ & $38 \%$ & $52 \%$ & $48 \%$ & $50 \%$ & $52 \%$ \\
\hline 7 & Brasil Escola & $42 \%$ & $71 \%$ & $71 \%$ & $46 \%$ & $19 \%$ & $43 \%$ & $31 \%$ & $52 \%$ \\
\hline 8 & Dr. Drauzio Varella & $39 \%$ & $70 \%$ & $63 \%$ & $33 \%$ & $34 \%$ & $49 \%$ & $42 \%$ & $49 \%$ \\
\hline 9 & $\begin{array}{l}\text { Secretaria Estadual de Saúde } \\
\text { do Paraná }\end{array}$ & $41 \%$ & $50 \%$ & $56 \%$ & $29 \%$ & $40 \%$ & $49 \%$ & $45 \%$ & $44 \%$ \\
\hline 10 & $\begin{array}{l}\text { Sociedade Brasileira de Pneu- } \\
\text { mologia e Tisiologia }\end{array}$ & $44 \%$ & $80 \%$ & $39 \%$ & $20 \%$ & $21 \%$ & $25 \%$ & $23 \%$ & $41 \%$ \\
\hline 11 & G1 (Globo) & $21 \%$ & $70 \%$ & $43 \%$ & $29 \%$ & $16 \%$ & $29 \%$ & $23 \%$ & $37 \%$ \\
\hline \multirow[t]{2}{*}{12} & $\begin{array}{l}\text { Fundação de Medicina Tro- } \\
\text { pical }\end{array}$ & $33 \%$ & $23 \%$ & $54 \%$ & $18 \%$ & $35 \%$ & $32 \%$ & $34 \%$ & $32 \%$ \\
\hline & Média & $42 \%$ & $68 \%$ & $63 \%$ & $36 \%$ & & & $44 \%$ & $50 \%$ \\
\hline
\end{tabular}

Um dos principais desafios do enfrentamento da tuberculose no Brasil é o abandono do tratamento. Para obter a cura, o tratamento é longo. Há uma série de motivos que dificultam sua adesão. Couto et al. (2014, P. 577) afirmam que:

Cabe aos profissionais de saúde transmitir as informações necessárias, apresentando ao usuário os possíveis efeitos colaterais decorrentes da ingestão da medicação, mas sempre enfatizando a importância de seguir o tratamento adequadamente e pelo tempo necessário. (COUTO ET AL., 2014, P. 577)

Assim, percebemos que a informação de qualidade pode contribuir não só para a prevenção dessa doença, seu diagnóstico, como também para uma eventual transmissão. Contribui para a adesão ao tratamento oferecido pelo SUS. E informação sobre saúde, hoje, não é oferecida apenas pelos profissionais de saúde; é produzida, disseminada e acessada cada vez mais na Internet.

Outro aspecto relevante do estudo refere-se ao papel dos avaliadores. De acordo com Paolucci (2015), a participação de 'usuários' e de 'especialistas' como avaliadores esteve presente em apenas 2,5\% dos estudos de avaliação de informação em sites de saúde. Nesses estudos, não foi encontrada relação nem padronização entre o número de participantes de cada grupo.

Paolucci (2015) identificou um caso digno de nota. Trata-se do trabalho desenvolvido por Montoya et al. (2013). Ele foi um dos poucos que contou com a participação de especialistas e usuários em seu processo avaliativo. Ao avaliar a qualidade da informação em dez sites de Transtorno de Atenção e Hiperatividade (TDAH), os autores envolveram 24 mães e onze pais de filhos com esse transtorno. Além disso, convidaram onze psiquiatras infantis e cinco neurologistas 
infantis que haviam participado de experimentos clínicos em TDAH e voluntariamente aceitaram participar do desenho e da implantação desta pesquisa. Dentre os pais, $70 \%$ possuíam formação de nível superior e $80 \%$ estavam na faixa etária de 30 a 50 anos (MONTOYA ET AL., 2013). Na Espanha, país em que o estudo foi realizado, apenas $34 \%$ das mulheres e $31 \%$ dos homens adultos têm essa formação (INE, 2012). Nesse sentido, a amostra de 'usuários' avaliadores utilizada por Montoya et al. (2013) não representa a maioria da população espanhola nesta faixa etária.

A experiência de Montoya et al. (2013) difere da relatada neste artigo, porque aqui os pesquisadores cidadãos envolvidos podem ser considerados representantes dos usuários do SUS. Os profissionais de saúde representaram os especialistas em tuberculose. Os dois grupos de avaliadores tiveram, ainda, um número aproximado de participantes. Na experiência descrita e analisada por Montoya et al. (2013), há maior disparidade entre os dois grupos: os usuários totalizaram 35 avaliadores; os profissionais, 16. Entretanto, uma limitação pode ser identificada nas duas experiências: o grupo de avaliadores envolvidos com a experiência relatada neste artigo não é suficientemente heterogêneo para abarcar o conjunto de possibilidades de usuários que acessam a Internet em busca de informação sobre patologias. No caso da tuberculose, a equipe poderia ter envolvido cidadãos acometidos com tuberculose, seus parentes e vizinhos.

O terceiro aspecto dessa experiência está relacionado aos critérios e indicadores de qualidade empregados. Foram utilizados simultaneamente os cinco critérios sugeridos por Eysenbach et al. (2002). Esse esforço não esteve presente em nenhum dos estudos analisados nas duas revisões sistemáticas mencionadas (EYSENBACH ET AL., 2002; PAOLUCCI, 2015). Nenhuma das iniciativas internacionais de avaliação contemplou os cinco critérios em seus instrumentos de avaliação (PAOLUCCI, 2015).

Além disso, a experiência relatada neste artigo se deu graças à participação dos pesquisadores cidadãos e de profissionais de saúde. Foram eles que construíram os indicadores durante um processo de reflexão e criação coletiva. Essa equipe foi capaz de criticar, construir e modificar indicadores.

Cabe destacar uma diferença identificada entre os dois trabalhos de avaliação realizados pelo LaISS - o primeiro com as informações de dengue e este com informações de tuberculose. No primeiro trabalho, a redação dos indicadores de 'acurácia' esteve estruturada em forma de perguntas (PEREIRA NETO; PAOLUCCI, 2014). Um dos a ser oferecido neste sentido é o seguinte: "A informação sobre prevenção está de acordo com o atual estágio do conhecimento científico?" (PEREIRA NETO; PAOLUCCI, 2014, P. 76). Esta pergunta pressupõe que o profissional conheça a resposta, que ele esteja atualizado em relação ao diagnóstico e ao tratamento da tuberculose. No caso da tuberculose, foi adotada estratégia distinta: a avaliação da 'acurácia' da informação de tuberculose na Internet estruturou-se sob a forma de afirmações e não de perguntas. Por exemplo, em relação à prevenção da tuberculose, pretendia-se verificar se o site continha a seguinte informação: 'A tuberculose pode ser prevenida em lugares arejados'. As opções de respostas para esse indicador eram 'Completa', 'Incompleta', 'Ausente' e 'Incorreta'. Ao utilizar sentenças afirmativas, a equipe da pesquisa convida o avaliador a verificar se a informação está completa ou não, ausente ou errada. Essa estrutura permitiu que os pesquisadores cidadãos avaliassem esse critério, situação que não ocorreu na avaliação de informações sobre dengue (PEREIRA NETO; PAOLUCCI, 2014).

Ficamos interessados em saber se as respostas ao critério acurácia variaram muito entre os dois grupos de avaliadores. A tabela 2, ilustrada a seguir, revela que o 'coeficiente de correlação' da avaliação realizada por 'usuários e especialistas' ficou acima de 0,82 na maioria dos sites (nove). Nos três sites em que o 'coeficiente' foi menor do que 0,82 , 
foram obtidos os coeficientes 0,79, 0,76 e 0,52. Esses dados revelam que houve associação entre as duas perspectivas de avaliadores no julgamento da conformidade das informações existentes nos sites de tuberculose. Apesar de a avaliação dos pesquisadores cidadãos terem resultado, na maioria dos casos, em percentuais de conformidade menores do que os da avaliação dos profissionais de saúde, podemos afirmar que o julgamento dos 'usuários' foi coerente com o julgamento dos 'especialistas'. Independentemente do grupo, cabe ressaltar que as respostas dos avaliadores aos indicadores de qualidade dependem de suas percepções. Um avaliador pode não ter encontrado uma determinada informação que esteja disponível no site. Contudo, se poucos avaliadores foram capazes de encontrá-la, então pode ser necessário que o gestor do site apresente a informação de outra forma.

Tabela 2. Ranking pelo critério 'Acurácia'

\begin{tabular}{|c|c|c|c|c|c|}
\hline \multirow{2}{*}{ Posição } & \multirow{2}{*}{ Site } & \multicolumn{4}{|c|}{ Acurácia } \\
\hline & & Usuários & Especialistas & Média & Correlação \\
\hline 1 & Programa Nacional de Controle de Tuberculose & $79 \%$ & $69 \%$ & $74 \%$ & 0,82 \\
\hline 2 & Secretaria Estadual de Saúde de São Paulo & $72 \%$ & $71 \%$ & $72 \%$ & 0,52 \\
\hline 3 & Prefeitura de Porto Alegre & $52 \%$ & $48 \%$ & $50 \%$ & 0,83 \\
\hline 4 & Prefeitura do Rio de Janeiro & $43 \%$ & $51 \%$ & $47 \%$ & 0,90 \\
\hline 5 & Wikipédia & $31 \%$ & $60 \%$ & $46 \%$ & 0,82 \\
\hline 6 & Secretaria Estadual de Saúde do Paraná & $40 \%$ & $49 \%$ & $45 \%$ & 0,86 \\
\hline 7 & Minha Vida & $29 \%$ & $55 \%$ & $42 \%$ & 0,82 \\
\hline 8 & Dr. Drauzio Varella & $34 \%$ & $49 \%$ & $42 \%$ & 0,76 \\
\hline
\end{tabular}

O último aspecto que destacamos nesta discussão diz respeito à avaliação com o critério de 'legibilidade', que propõe a verificação do nível de compreensão da informação, ou seja, se ela é fácil ou difícil de ser entendida. De acordo com Paolucci (2015), esse foi o segundo critério mais utilizado nos estudos de avaliação de qualidade de informação em sites de saúde realizados internacionalmente entre 2001 e 2014. A maioria utilizou ferramentas de avaliação de legibilidade como Flesch-Kincaid Grade Level, Flesch Reading Ease e Gunning Fog Index. A revisão sistemática de Eysenbach et al. (2002) concluiu que o critério não tinha sido avaliado pelos reais consumidores da informação. O trabalho de Paolucci (2015) mostrou que essa lacuna continua existindo, apesar de três estudos terem contado com a participação de usuários (GLENTON; NILSEN; CARLSEN, 2006; LEVEQUE ET AL., 2007; NICOLSON ET AL., 2011). Assim a maioria dos estudos de avaliação da qualidade da informação em sites de saúde (94\%) utilizam atualmente programas de computador que usam fórmulas matemáticas para calcular o nível de 'legibilidade' de textos escritos (PAOLUCCI, 2015). Esses processos de avaliação têm limitações, porque:

não refletem outros fatores que afetam a compreensão como frequência e explicação de jargão médico, estilo de escrita (uso de voz ativa, linguagem não paternalista, mensagens motivacionais, tom/humor, como se relaciona com o público), ou uso de informação culturalmente específica. (EYSENBACH ET AL., 2002, P. 2694). 
Um dos aspectos que singulariza o trabalho relatado neste artigo foi a participação de usuários do SUS como avaliadores de 'legibilidade’ das informações em sites de tuberculose. Esse público pode ser considerado representante dos reais consumidores da informação, pois a tuberculose é um problema enfrentado no território em que residem. Não temos conhecimento de outra iniciativa nesse campo que tenha empreendido esforço semelhante.

\section{Considerações finais}

A experiência relatada neste artigo guarda certa singularidade por ter contado com a participação de usuários e profissionais.

No Brasil, não foram identificados estudos que tenham desenvolvido pesquisa participativa em avaliação da qualidade da informação disponível em sites de saúde, apesar de a produção acadêmica sobre o tema ter crescido (PAOLUCCI, 2015). Enquanto isso, agências governamentais, associações profissionais e pesquisadores têm feito avaliação de informação em sites de saúde há cerca de 20 anos, principalmente nos Estados Unidos e na Europa. O campo de avaliação de informações em sites de saúde está em expansão e há preocupação com a qualidade da informação veiculada na Internet.

Por fim, entendemos ser interessante fazer duas recomendações para os interessados em desenvolver pesquisas futuras neste campo.

Cabe destacar, inicialmente, que não temos conhecimento de um instrumento de avaliação da qualidade da informação disponível na Internet que contemple os cinco critérios e que tenha sido construído por meio de técnicas psicométricas. Essas técnicas visam a medir os processos mentais de indivíduos para entender o sentido de suas respostas, por exemplo, a um questionário com vários itens (PASQUALI, 2009). Segundo esse autor, os testes psicométricos possuem dois principais parâmetros para seu reconhecimento como legítimos: 'validade' e 'precisão'. A 'validade' seria relacionada, por exemplo, ao fato de um questionário medir o que se espera. A 'precisão' diz respeito a uma medição com o mínimo possível de erros. Além disso, os resultados obtidos devem ser os mesmos em testes realizados em momentos diferentes com grupos semelhantes de indivíduos. As técnicas psicométricas, portanto, permitem validar um instrumento que, de fato, meça o construto da qualidade da informação, seja reconhecido como apropriado para essa finalidade e possa ser reutilizado em diferentes momentos.

Outro aspecto a ser mencionado refere-se à definição de Eysenbach et al. (2002, P. 2695) para o critério 'acurácia'. Segundo esses autores, tal critério pretende medir o "grau de concordância da informação oferecida com a melhor evidência ou com a prática médica geralmente aceita”. O grau de concordância da informação com a prática médica é realizado, geralmente, por meio do consenso de um número reduzido de especialistas. Esse processo não garante, obrigatoriamente, que o resultado do consenso esteja atual e correto (NADANOVSKY, 1999). Pelo contrário, os profissionais envolvidos podem estar desatualizados em relação às melhores evidências científicas. Eles podem, ainda, apresentar opiniões e condutas que estejam em desuso. Assim, as pesquisas de avaliação da qualidade das informações em sites de saúde devem construir indicadores de qualidade baseados em estudos de revisão sistemática, substituindo o consenso de especialistas por uma síntese do conhecimento proveniente da literatura científica.

Finalmente, apesar de algumas limitações, entendemos que este projeto, realizado pelo LaISS, guarda todas as condições de participar de forma crítica e criativa do debate internacional sobre os critérios de avaliação de sites de saúde. Como estudo piloto, com número reduzido de sites avaliados e com a participação limitada de avaliadores, torna-se arriscado validar as generalizações 
desta experiência. De qualquer forma, o projeto sugere que o problema da qualidade da informação de saúde on-line afeta sites públicos e privados. Este artigo pretende ressaltar tal esforço.

Este artigo e esta experiência pretendem colocar em debate a importância da certificação de sites como opção para orientar o público em geral e os profissionais de saúde sobre a qualidade da informação em saúde disponível na Internet. A Fundação Oswaldo
Cruz está discutindo a criação de um Selo Fiocruz de Qualidade de Informação em Sites de Saúde. A proposta consta do 'Portfólio de Inovação. Fundação Oswaldo Cruz. Inovação em saúde para a sociedade' da Vice-Presidência de Produção e Inovação em Saúde (VPPIS) da Fiocruz e conta com o apoio da Direção da Escola Nacional de Saúde Pública Sergio Arouca (Ensp/ Fiocruz).

\section{Referências}

AGRESTI, A.; FINLAY, B. Métodos estatísticos para as ciências sociais. 4. ed. Porto Alegre: Penso, 2012. 664 p.

BARBOSA, A. L.; MARTINS, E. N. Avaliação

da informação dos pacientes sobre miopsias e fotopsias através da Internet. Arquivos Brasileiros de Oftalmologia, São Paulo, v. 70, n. 5, p. 839-843, out. 2007.

BARBOSA, A. F. Pesquisa sobre o uso das tecnologias de informação e comunicação no Brasil: TIC Domicílios e Empresas 2013. São Paulo: Comitê Gestor da Internet no Brasil, 2014.

BORGES, E. D. Educação permanente em saúde: uma estratégia em construção para a gestão do programa saúde da família do Centro de Saúde Escola Germano Sinval Faria. 2007. 152 f. Dissertação (Mestrado em Gestão de Ciência \& Tecnologia em Saúde) - Escola Nacional de Saúde Pública Sergio Arouca, Fundação Oswaldo Cruz, Rio de Janeiro, 2007.

BRASIL. Ministério da Saúde. Secretaria de Vigilância em Saúde. Boletim Epidemiológico, v. 44, n. 2, Brasília,
DF, 2013. Disponível em: <http://portalsaude.saude.gov. br/images/pdf/2014/maio/06/boletim2-2013-tb-web. pdf>. Acesso em: 1 nov. 2016.

COUTO, D. S. et al. Fatores determinantes para o abandono do tratamento da tuberculose: representações dos usuários de um hospital público. Saúde em Debate, Rio de Janeiro, v. 38, n. 102, p. 572581 , set. 2014 .

CONSELHO REGIONAL DE MEDICINA DO ESTADO DE SÃO PAULO (CREMESP). Resolução. Disponível em: <http://www.cremesp.org.br/?siteAcao=Publicacoe sConteudoSumario\&id=25>. Acesso em: 26 abr. 2016.

CUBAS, M. R.; FELCHNER, P. C. Z. Análise das fontes de informação sobre os autoexames da mama disponíveis na Internet. Ciência \& Saúde Coletiva, Rio de Janeiro, v. 17, n. 4, p. 965-970, abr. 2012.

DEL GIGLIO, A. et al. Qualidade da informação da internet disponível para pacientes em páginas em português. Revista da Associação Médica Brasileira, São Paulo, v. 58, n. 6, p. 645-649, dez. 2012. 
EYSENBACH, G. et al. Empirical Studies Assessing the Quality of Health Information for Consumers on the World Wide Web: A Systematic Review. JAMA, Chicago, v. 287, n. 20, p. 2691-2700, 2002.

FERNANDES, T. M.; COSTA, R. G. As comunidades de Manguinhos na história das favelas no Rio de Janeiro. Revista Tempo, Niterói, v. 19, n. 34, p. 117-133, jan./jun. 2013.

FREIRE, P. Extensão ou comunicação? Rio de Janeiro: Paz e Terra, 1971.

FUREDI, F. The end of professional dominance. Society, New Brunswick, v. 43, n. 6, p. 14-18, set. 2006.

\section{GLENTON, C.; NILSEN, E. S.; CARLSEN, B. Lay}

perceptions of evidence-based information - a qualitative evaluation of a website for back pain sufferers. BMC health services research, Londres, n. 6, 2006.

GUARDIOLA-WANDEN-BERGHE, R.; SANZVALERO, J.; WANDEN-BERGHE, C. Quality assessment of the Website for Eating Disorders: a systematic review of a pending challenge. Ciência \&t Saúde Coletiva, Rio de Janeiro, v. 17, n. 9, p. 2489-2497, 2012.

INSTITUTO NACIONAL DE ESTADÍSTICA (INE). Nivel de estudios de la población adulta (de 25 a 64 años). Disponível em: <http://www.ine.es/ss/ Satellite?L=es_ES\&c=INESeccion_C\&cid $=12599254816$ $59 \& \mathrm{p}=\backslash$ \&pagename $=$ ProductosYServicios $\% 2$ FPYSLayo ut\&param3=1259924822888>. Acesso em: 13 fev. 2015.

LEVEQUE, M. et al. Evaluation of neuro-oncology information for French speaking patients on the Internet. Neurochirurgie, Paris, v. 53, n. 5, p. 343-355, nov. 2007.

LOPES, I. L. Iniciativas internacionais para o controle da qualidade da informação em Saúde na Web. RECIIS, Rio de Janeiro, v. 6, n. 2, 29 jun. 2012.

MENDES, A. M.; FENSTERSEIFER, L. M.

Tuberculose: porque os pacientes abandonam o tratamento? Bol. Pneumol. Sanit, Rio de Janeiro, v. 12, n. 1, p. 27-38, 2004.

MENDONÇA, A. P. B.; PEREIRA NETO, A. F. Critérios de avaliação da qualidade da informação em sites de saúde: uma proposta. RECIIS, Rio de Janeiro, v. 9, n. 1, jan./mar. 2015.

MONTOYA, A. et al. Evaluating Internet information on attention-deficit/hyperactivity disorder (ADHD) treatment: parent and expert perspectives. Education for health (Abingdon), Abingdon, v. 26, n. 1, abr. 2013.

NADANOVSKY, P. Epidemiologia aplicada a clínica: um enfoque científico do uso da informação médica: Medicina Baseada em Evidência. Rio de Janeiro: Uerj, 1999. p. 1-27. (Estudos em Saúde Coletiva, v. 185).

NICHOLSON, L. et al. Quality of internet-derived information on common ophthalmic conditions. The British journal of ophthalmology, Londres, v. 98, n. 3, p. 420, mar. 2014.

OLIVEIRA, M. D.; OLIVEIRA, R. D. Pesquisa Social e Ação Educativa: Conhecendo a Realidade Para Poder Transformá-la. In: BRANDÃO, C. R. (Org.). Pesquisa Participante. São Paulo: Brasiliense, 1985. p. 83-95.

PAOLUCCI, R. Métodos para avaliação da qualidade de informação em sites de saúde: revisão sistemática (20012014). 2015. 82 f. Dissertação (Mestrado em Ciências) - Instituto de Comunicação e Informação Científica e Tecnológica em Saúde, Fundação Oswaldo Cruz, 2015.

PASQUALI, L. Psicometria. Rev. esc. enferm. USP, São Paulo, v. 43, n. esp., p. 992-999, dez. 2009.

PASSOS, E. et al. O Comitê Cidadão como estratégia cogestiva em uma pesquisa participativa no campo da saúde mental. Ciência \&t saúde coletiva, Rio de Janeiro, v. 18, n. 10, p. 2919-2928, 2013.

PEREIRA NETO, A. F. et al. Avaliação de sites de saúde em questão: a Aids nos sites brasileiros de Organizações Não Governamentais (ONG) de Lésbicas, Gays, Bissexuais, Travestis e Transexuais (LGBT). RECIIS, v. 7, n. 1, mar. 2013. Disponível em: <http:// 
www.reciis.icict.fiocruz.br/index.php/reciis/article/

view/451>. Acesso em: 7 nov. 2016.

PEREIRA NETO, A. F.; PAOLUCCI, R. Qualidade

da informação em sites de dengue: análise de uma

experiência inovadora. Rio de Janeiro: Ensp, 2014.

SILVA, R. Q.; GUBERT, M. B. Qualidade das

informações sobre aleitamento materno e alimentação complementar em sites brasileiros de profissionais de saúde disponíveis na internet. Revista Brasileira de Saúde Materno Infantil, Recife, v. 10, n. 3, p. 331-340, set. 2010.

SILVEIRA, P. C. M.; COSTA, A. E. S.; LIMA, C. C. Gagueira na web: qualidade da informação. Revista CEFAC, Campinas, v. 14, n. 3, p. 430-437, jun. 2012.
SOUZA, C. L. N.; LUZ, Z. P.; RABELLO, A. Análise da informação sobre a leishmaniose visceral disponível em portais brasileiros da rede mundial de computadores: internet. Revista da Sociedade Brasileira de Medicina Tropical, Uberaba, v. 41, n. 4, p. 352-357, ago. 2008.

WIKIRIO. IDH dos bairros da cidade do Rio de Janeiro. Disponível em: <http://www.wikirio.com.br/IDH_dos_ bairros_da_cidade_do_Rio_de_Janeiro>. Acesso em: 14 abr. 2016.

Recebido para publicação em abril de 2016

Versão final em outubro de 2016

Conflito de interesses: inexistente

Suporte financeiro: não houve 